University of Nebraska - Lincoln

DigitalCommons@University of Nebraska - Lincoln

$11-28-2003$

\title{
Laser-Energy Transfer and Enhancement of Plasma Waves and Electron Beams by Interfering High-Intensity Laser Pulses
}

\author{
P. Zhang \\ University of Michigan, Ann Arbor \\ N. Saleh \\ University of Michigan, Ann Arbor \\ Shouyuan Chen \\ University of Michigan-Ann Arbor, schen6@unl.edu \\ Z.M. Sheng \\ Laboratory of Optical Physics, Institute of Physics, People's Republic of China \\ Donald P. Umstadter \\ University of Nebraska-Lincoln, donald.umstadter@unl.edu
}

Follow this and additional works at: https://digitalcommons.unl.edu/physicsumstadter

Part of the Physics Commons

Zhang, P.; Saleh, N.; Chen, Shouyuan; Sheng, Z.M.; and Umstadter, Donald P., "Laser-Energy Transfer and Enhancement of Plasma Waves and Electron Beams by Interfering High-Intensity Laser Pulses" (2003). Donald Umstadter Publications. 21.

https://digitalcommons.unl.edu/physicsumstadter/21

This Article is brought to you for free and open access by the Research Papers in Physics and Astronomy at DigitalCommons@University of Nebraska - Lincoln. It has been accepted for inclusion in Donald Umstadter Publications by an authorized administrator of DigitalCommons@University of Nebraska - Lincoln. 


\title{
Laser-Energy Transfer and Enhancement of Plasma Waves and Electron Beams by Interfering High-Intensity Laser Pulses
}

\author{
P. Zhang, ${ }^{1}$ N. Saleh, ${ }^{1}$ S. Chen, ${ }^{1}$ Z. M. Sheng, ${ }^{2}$ and D. Umstadter ${ }^{1}$ \\ ${ }^{1}$ FOCUS Center, University of Michigan, Ann Arbor, Michigan 48109, USA \\ ${ }^{2}$ Laboratory of Optical Physics, Institute of Physics, P.O. Box 603, Beijing 100080, People's Republic of China
}

(Received 14 February 2003; published 25 November 2003)

\begin{abstract}
The effects of interference due to crossed laser beams were studied experimentally in the highintensity regime. Two ultrashort (400 fs), high-intensity $\left(4 \times 10^{17}\right.$ and $\left.1.6 \times 10^{18} \mathrm{~W} / \mathrm{cm}^{2}\right)$ and $1 \mu \mathrm{m}$ wavelength laser pulses were crossed in a plasma of density $4 \times 10^{19} \mathrm{~cm}^{3}$. Energy was observed to be transferred from the higher-power to the lower-power pulse, increasing the amplitude of the plasma wave propagating in the direction of the latter. This results in increased electron self-trapping and plasma-wave acceleration gradient, which led to an increased number of hot electrons (by 300\%) and hot-electron temperature (by 70\%) and a decreased electron-beam divergence angle (by $45 \%$ ), as compared with single-pulse illumination. Simulations reveal that increased stochastic heating of electrons may have also contributed to the electron-beam enhancement.
\end{abstract}

DOI: 10.1103/PhysRevLett.91.225001

PACS numbers: 52.38.Kd, 41.75.Jv, 52.57.- $\mathrm{z}$

The interactions of high-intensity laser pulses in plasma are of much current interest because of their relevance to advanced radiation sources, inertial fusion concepts, and accelerators [1]. These interactions often take the form of intersecting beams, in which case several novel physical phenomena are expected to occur. For instance, when an intense laser pulse interacts with a solid-density target, it is partially reflected, and the beating between the incident and reflected light in the underdense plasma region will create interference, which can modify the optical properties of the plasma [2], excite plasma waves [3], and heat electrons [4]. It has been suggested theoretically [5] that this stochastic heating of electrons is ultimately responsible for the acceleration of multi-MeV energy beams of ions from laser-irradiated thin foils [6-8]. In inertial fusion research, the electron heating and modification of the laser reflectivity that results from crossed laser beams [9-12] must be controlled in order to efficiently couple energy to the fusion target. High-intensity crossed beams are relevant to fastignition fusion research [13]. The overlapping of intense laser pulses in plasmas has also been suggested theoretically [14-16] as one of several means [14-19] to optically inject electrons into laser-driven plasma waves.

Reported here are experimental results on crossedbeam interference in the high-intensity (relativisticelectron) regime and its effects on the dielectric and thermal properties of a plasma. Energy transfer between the two laser beams as well as plasma-wave and electronbeam enhancement are observed. The mechanism responsible for the energy transfer in this case differs from that studied in previous long-pulse and low-power crossedbeam experiments [9-12]. In our study, the mechanism was scattering from a stationary electron-density grating that was driven directly by optical interference, while in these previous experiments it was scattering from ion waves. The physics of overlapping short-duration pulses in plasmas were previously studied theoretically [2-4]. For instance, plasma-wave excitation [3] and energy transfer by superradiant amplification of a short pulse by a counterpropagating pulse [2] are predicted to occur when the difference in frequency between the two pulses is equal to the plasma frequency. We studied the degenerate case, where the frequency difference is zero, which closely approximates the interference that occurs when light is reflected at its critical density.

The experiments were performed with a hybrid Ti:sapphire and Nd:glass laser system, delivering short pulses $(400 \mathrm{fs}, 1.053 \mu \mathrm{m})$ in a single shot, with power up to $10 \mathrm{TW}$. The laser beam was split into two beams, one of which contained $80 \%$ of the laser energy (injection) and the other one $20 \%$ (pump). These two beams were focused with two $f / 3$ parabolic mirrors to nearGaussian vacuum spot sizes of $12 \mu \mathrm{m}$ FWHM (full width at half maximum), which contained $60 \%$ of the total energy. This corresponds to focal intensities ranging from $\left(3 \times 10^{17}\right)-\left(5 \times 10^{17}\right) \mathrm{W} / \mathrm{cm}^{2}$ for pump beam and $\left(1.2 \times 10^{18}\right)-\left(2 \times 10^{18}\right) \mathrm{W} / \mathrm{cm}^{2}$ for injection. The two beams of the parallel polarization were perpendicularly overlapped onto the edge of a supersonic helium gas jet. The pressure of the helium gas was 800 psi and the gas was fully photoionized, creating a plasma with density that was $4 \times 10^{19} / \mathrm{cm}^{3}$, corresponding to $n_{0} / n_{c}=0.04$, where $n_{c}$ is the critical density.

The laser powers for both pump and injection pulses were above $1 \mathrm{TW}$, higher than the critical power for relativistic self-focusing, given by $P=17\left(\omega_{0} / \omega_{p}\right)^{2} \mathrm{GW}$, where $\omega_{0}$ and $\omega_{p}$ are the laser and plasma frequencies, respectively. The pulse durations ( $\tau=400 \mathrm{fs}$ ) were many plasma periods long $\tau>50\left(2 \pi / \omega_{p}\right)$. In previous experiments performed with similar parameters [20], plasma waves were observed to be driven by the self-modulated 
wakefield mechanism [21-23]. Images of crossed plasma channels were observed with top and side imaging of Thomson scattering and recorded by a CCD camera. Spatial profiles of pump electron beam were recorded with a scintillating screen (LANEX) coupled to a CCD camera. The LANEX screen was blocked by two layers of Al foils each of the thickness of $40 \mu \mathrm{m}$, which blocked electrons whose kinetic energies were below $170 \mathrm{keV}$. Pump electron spectra were obtained with an electron spectrometer $(260 \mathrm{keV}-5 \mathrm{MeV})$, composed of a dipole permanent magnet with a LANEX screen imaged by a CCD camera. In this case, a single aluminum foil of $40 \mu \mathrm{m}$ thickness in front of the screen blocked the laser light. A 1-mm wide slit was placed in front of the magnet in order to select a narrow range of angles for electrons entering the magnet. The distance between the slit and the gas jet was $30 \mathrm{~mm}$. Spatial and temporal overlap was achieved by adjusting the positions and delay between the beams until a bright spot in the intersection of the two plasma channels was observed. The bright spot was due to enhanced Thomson scattering [24] by the density bunched in the interference valleys.

Figure 1 shows the spatial profiles of the pumpaccelerated electrons of the kinetic energy above $170 \mathrm{keV}$ at laser power of $1.5 \mathrm{TW}$. It can be seen that with injection a more collimated and intensified electron jet was found (right inset), with a beam divergence angle of about $7^{\circ}$ (the angle corresponding to the FWHM of the electronbeam profile), while without injection (right inset) it was nearly $12^{\circ}$. Integration of the intensity distributions

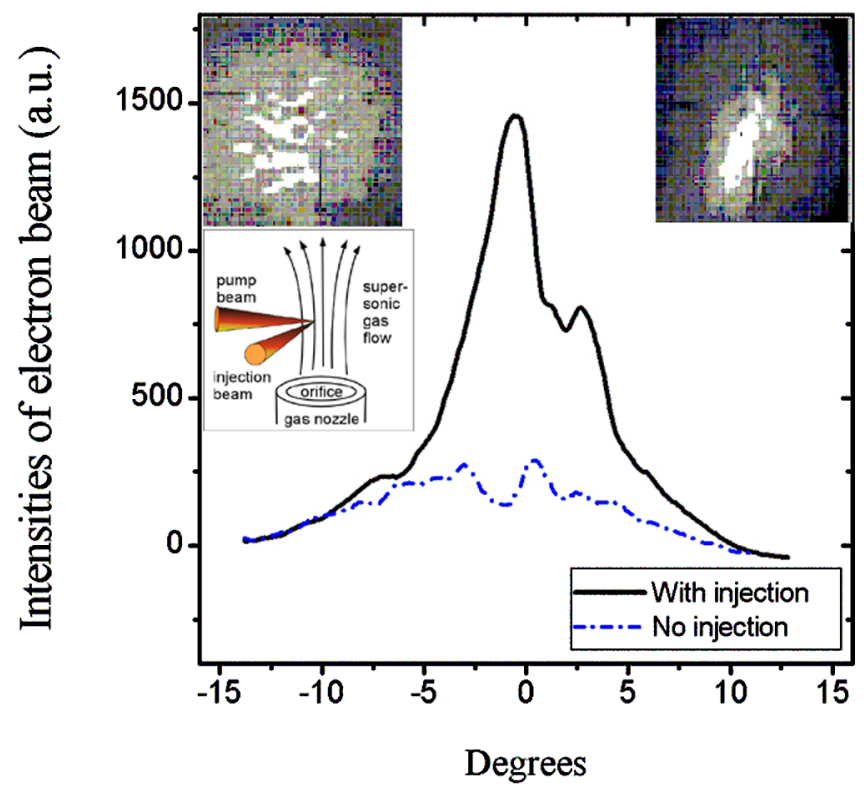

FIG. 1 (color online). Profiles of the spatial distributions of the electron beams (kinetic energies above $170 \mathrm{keV}$ ) in the direction of the pump at pump laser power of $1.5 \mathrm{TW}$, with (right inset) and without injection (left inset). Schematic diagram of the laser beams intersecting in the gas flow (middle left inset). showed that, with injection, the charge of electronbeam was increased $\sim 3$ times.

In the conditions of best laser pulse temporal overlap, when the time delay between pump and injection was $\pm 30 \mathrm{fs}$, and with a pump laser power of $1.05 \mathrm{TW}$, the electron spectra in the direction of the pump, with and without injection, are as shown in Fig. 2. With injection, it can be seen that the portion of the electrons with kinetic energies greater than $400 \mathrm{keV}$ is enhanced. As in previous experiments [20], the electron spectra approximated a Maxwellian-like distribution, i.e., $\exp \left(-\epsilon_{k} / T_{e}\right)$ with a high-energy tail, where $\epsilon_{k}$ is the electron kinetic energy and $T_{e}$ is the electron temperature. The corresponding electron temperatures were fitted and shown in Fig. 2, and an increase of electron temperature with injection was observed to be more than $60 \%$. Figure 3 shows the temperatures fitted from the electron spectra, with or without injection, at different laser powers. When injection was on, the increase of electron temperature was between $30 \%$ and $70 \%$ and corresponds roughly to the depth of the ponderomotive potential well due to the interference between the two pulses. In the high-energy tails, the temperature difference was only about $10 \%$, even though the relative electron number was higher with injection.

Figure 4 shows the spectra of light exiting the plasma in the pump direction, with and without injection. With dual-beam illumination, the light signals in a broad spectrum range were obviously increased indicating that energy was transferred from the high-power to the low-power beam. It can also be seen from Fig. 3 that a $20 \%$ increase in pump power results in an electron temperature and number to levels that are nearly the same as

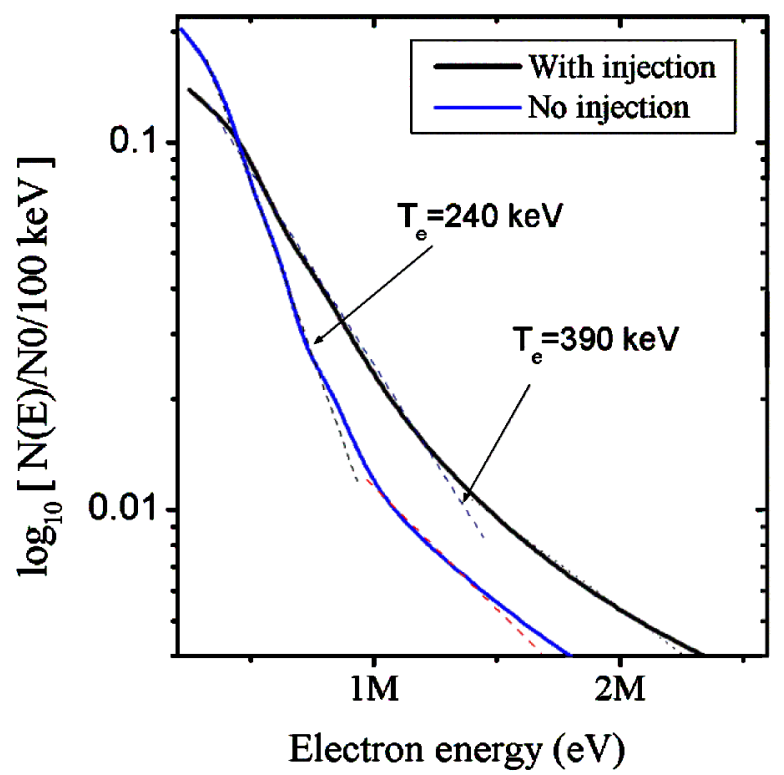

FIG. 2 (color online). The energy spectra of pump electron beam at pump laser power $1.05 \mathrm{TW}$, with (top curve) and without (bottom curve) injection. Dotted lines are exponential fits. The higher energy tails are $600 \mathrm{keV}$ without injection and $660 \mathrm{keV}$ with injection. 


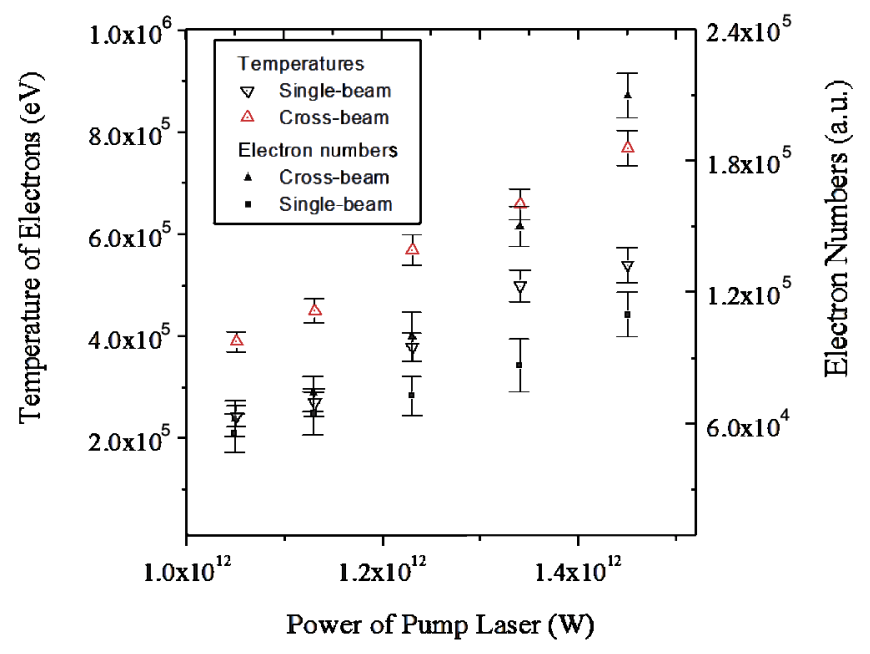

FIG. 3 (color online). The dependence of the electron temperature and number on the power of the pump laser, with and without injection.

those achieved by the addition of the injection pulse without increasing the pump.

A two-dimensional particle-in-cell (PIC) code computer simulation was also run with parameters similar to those of the experiment. A rectangular simulation box of $160 \lambda \times 60 \lambda$ was used, which was split into many cells, each with a size of $1 / 10 \lambda \times 1 / 10 \lambda$ for the integration of Maxwell's equations. A homogeneous plasma volume with an initial density of $0.04 n_{c}$ occupied part of the simulation box. The pump pulse along the $x$ direction and the injection pulse along the $y$ direction initially were $a_{10}=0.5$ and $a_{20}=1.0$, respectively. Nine particles per cell were used for electrons and ions. Absorption boundaries for the fields and reflection boundaries for particles were used both in the $x$ and the $y$ directions.

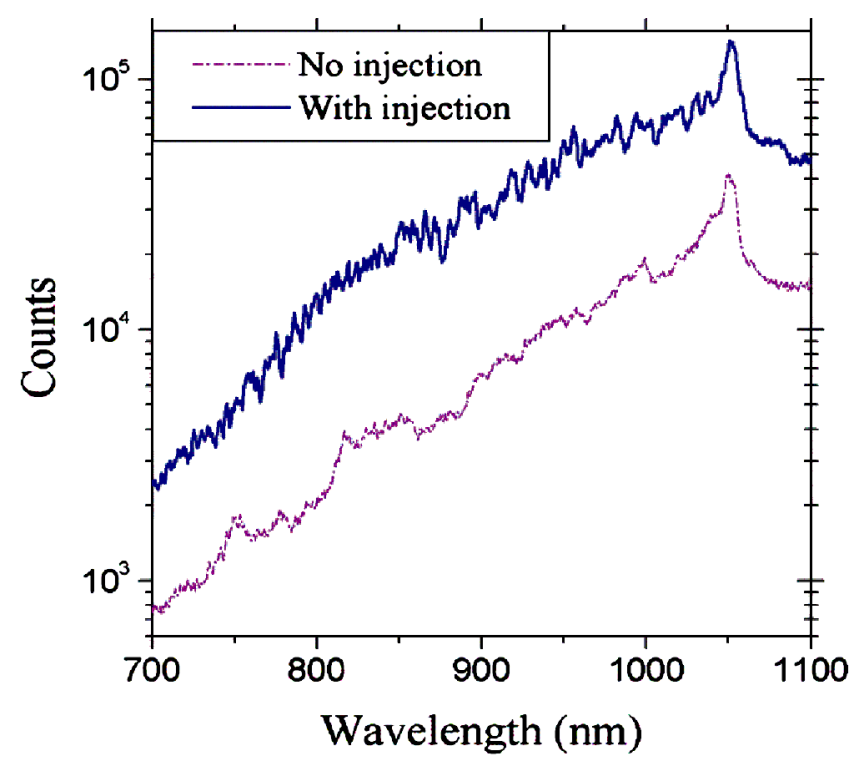

FIG. 4 (color online). The light spectra in the pump direction, with and without injection.
The results show that, in the region of laser intersection, electrons were trapped in the potential valleys by the ponderomotive force of the laser interference, resulting in the formation of a density grating with peak density modulation $n>10 n_{0}$, where $n_{0}$ was the background density. This same density bunching was predicted by balancing the ponderomotive force with the electrostatic force and electron thermal pressure. It was this density modulation that enhanced the scattering [24] observed in the experiments. The potential of ponderomotive force $\phi_{p}=(\gamma-1) m_{0} c^{2}$, where $\gamma=\sqrt{1+a_{0}^{2} / 2}$ was the relativistic factor, $a_{0}=8.5 \times 10^{-10} \lambda[\mu \mathrm{m}] I^{1 / 2}\left[\mathrm{~W} / \mathrm{cm}^{2}\right]$ was the normalized vector potential, and $\lambda$ and $I$ were the laser wavelength and focal intensity, respectively. At the peak intensity of laser interference, $I=4 \times$ $10^{18} \mathrm{~W} / \mathrm{cm}^{2}$, the potential depth of the ponderomotive force valleys was estimated to be about $300 \mathrm{keV}$.

Figure 5(a) shows snapshots of the pump laser field, the electric field of the pump plasma wave, and the momentum of electrons at $t=100$ laser cycles. The two laser pulses fully overlapped at $t=80$ laser cycles. It can be seen that, during the time when the beams crossed, the velocities of electrons were increased to close to the speed of light in both positive and negative directions, indicating that the electrons were heated isotropically. Calculations indicate that the ponderomotive force of the injection pulse could increase the electron kinetic energy in the direction of the pump by $200 \mathrm{keV}$. But this might also be explained by stochastic motion of electrons in the region of laser intersection [4]. The calculated Liapunov exponent of the electron trajectory indicates that the amplitudes of pump and injection laser were sufficient to trigger stochastic motion. In addition, the plasma disturbance of the high density gradient (scale length of $\sim 0.7 \lambda$ and $n_{e} / n_{0} \sim 10$ ) excited by laser interference does create ideal conditions for stochastic heating of electrons. With these preheated electrons injected in the plasma wave in a broad range of phases, the number increase of high-energy electrons is to be expected.

In order to investigate the mechanism for electron heating and distinguish between the effects of laser power increase due to energy transfer and the role of stochastic heating, we did additional simulations under conditions of both dual-beam and single pump beam illumination, but in the latter case with a $20 \%$ power increase [Fig. 5(b)]. With injection, electrons of the kinetic energies less than $1 \mathrm{MeV}$ are obviously increased. With 400-fs injection laser pulses, electrons are injected into the self-modulated plasma wave at all phases. In the relatively short acceleration distance in the simulation $(\sim 100 \mu \mathrm{m})$, these initial velocity changes are obvious in the spectrum, while the maximum electron kinetic energy depends on the strength of the plasma wakefield, which was proportional to the laser power. The reason why the injection effects were not obvious in the results (Fig. 3) is that the optical injection happens only in the laser beam intersection over 

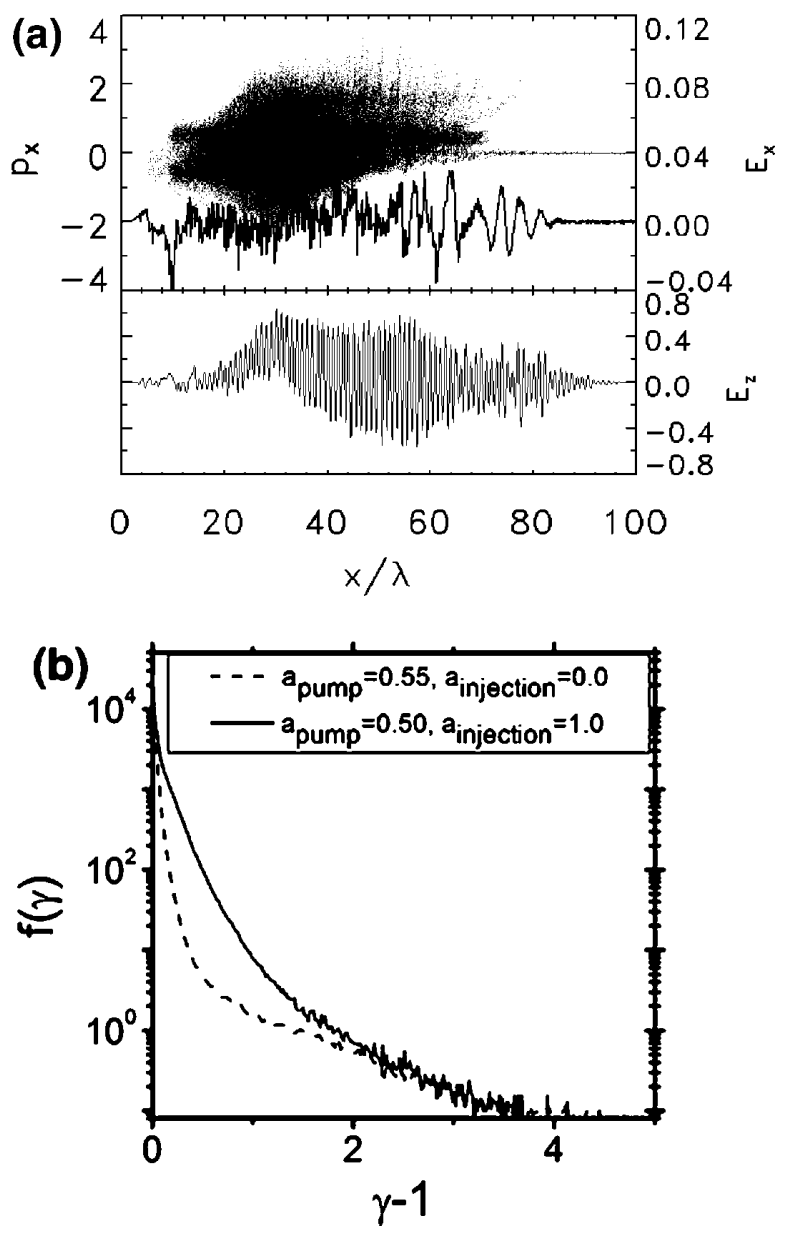

FIG. 5. (a) Simulation result showing snapshots of the pump laser field, the electric field of the pump plasma wave, and the momentum of electrons at $t=100$ laser cycles. (b) Simulated electron spectrum under conditions of both dual-beam and single pump beam illumination, but in the latter case with a $20 \%$ power increase of the weaker beam.

a scale length of $\sim 10 \mu \mathrm{m}$, while the distance of plasmawave propagation is nearly $1 \mathrm{~mm}$, about 100 times longer. Along the path of propagation, the self-modulated plasma wave continues to self-trap electrons over a large range of phases and accelerates them.

In summary, the first controlled experimental study of crossed-beam interference in the weakly relativistic regime was discussed. When the two high-intensity laser pulses were overlapped with each other in an underdense and uniform plasma, significant energy exchange from the higher-power to the lower-power laser beam was observed. The charge, temperature, and divergence angle of the electron beam that was accelerated in the direction of the lower-power laser beam were found to dramatically change with dual-laser-pulse illumination. These effects are unavoidable in current experiments on ion acceleration and fast-ignition fusion and in future experiments on laser-plasma accelerators. In the latter case, simulations [14-19] indicate that, through the ponderomotive force, one laser pulse can inject electrons into the plasma wave driven by the other laser pulse. When the pulse durations are equal to a plasma period, rather than many plasma periods, as in our experiment, and the plasma-wave amplitude is kept below the self-trapping threshold, this injection mechanism can reduce the electron-beam energy spread to just a few percent (as compared with $100 \%$ currently), rendering laser-wakefield accelerators more practical for applications in nuclear physics research, $\mathrm{x}$-ray free-electron lasers, and medicine. This study suggests that the effects of energy transfer must be considered and the mechanism of stochastic heating might also be used for optical injection. However, laser-plasma parameters that differ from those used in our experiment must be carefully chosen in order to inject electrons over a narrow range of phases, as required to produce monoenergetic electron beams.

The authors acknowledge the support of Division of High-Energy Physics, Office of Science, U.S. Department of Energy, Award No. DE-FG02-98ER41071, with lasers supported by the National Science Foundation FOCUS Center, Grant No. 0114336. We also thank G. Shvets, Y. Sentoku, and K. Mima for many useful discussions, and A. Maksimchuk for valuable assistance with the laser.

[1] D. Umstadter, Phys. Plasmas 8, 1774 (2001).

[2] G. Shvets, N. J. Fisch, and J.-M. Rax, Phys. Rev. Lett. 80, 2598 (1998).

[3] G. Shvets, N. J. Fisch, and A. Pukhov, Phys. Plasmas 9, 2383 (2002).

[4] Z.-M. Sheng et al., Phys. Plasmas 9, 3147 (2002).

[5] Y. Sentoku et al., Appl. Phys. B 74, 207 (2002).

[6] E. L. Clark et al., Phys. Rev. Lett. 84, 670 (2000).

[7] A. Maksimchuk et al., Phys. Rev. Lett. 84, 4108 (2000).

[8] R. A. Snavely et al., Phys. Rev. Lett. 85, 2945 (2000).

[9] R. K. Kirkwood et al., Phys. Rev. Lett. 76, 2065 (1996).

[10] K. B. Wharton et al., Phys. Rev. Lett. 81, 2248 (1998).

[11] C. Labaune et al., Phys. Rev. Lett. 85, 1658 (2000).

[12] R. K. Kirkwood et al., Phys. Rev. Lett. 89, 215003 (2002).

[13] M. Tabak et al., Phys. Plasmas 1, 1626 (1994).

[14] D. Umstadter, J. K. Kim, and E. Dodd, Phys. Rev. Lett. 76, 2073 (1996).

[15] E. Esarey et al., Phys. Rev. Lett. 79, 2682 (1997).

[16] R. G. Hemker et al., Phys. Rev. E 57, 5920 (1998).

[17] B. Rau, T. Tajima, and H. Hojo, Phys. Rev. Lett. 78, 3310 (1997).

[18] S. V. Bulanov et al., Plasma Phys. Rep. 25, 701 (1999).

[19] C. Moore et al., Phys. Rev. Lett. 82, 1688 (1999).

[20] D. Umstadter et al., Science 273, 472 (1996).

[21] P. Sprangle, A. Ting, E. Esarey, and A. Fisher, J. Appl. Phys. 72, 5032 (1992).

[22] T. M. Antonsen, Jr. and P. Mora, Phys. Rev. Lett. 69, 2204 (1992).

[23] N. E. Andreev et al., JETP Lett. 55, 571 (1992).

[24] R. E. Slusher and C. M. Surko, Phys. Fluids 23, 472 (1980). 